\title{
Identification of an immuno-dominant protein from Klebsiella pneumoniae strains causing pyogenic liver abscess: implication in serodiagnosis
}

Tzu-Lung Lin'1, Yi-Ping Chuang ${ }^{2}$, Yu-Tsung Huang ${ }^{3}$, Pei-Fang Hsieh¹, Yi-Tsung Lin ${ }^{4,5}$ and Jin-Town Wang 1,6*

\begin{abstract}
Background: Klebsiella pneumoniae has emerged worldwide as a cause of pyogenic liver abscess (PLA) often complicated by meningitis and endophthalmitis. Early detection of this infectious disease will improve its clinical outcome. Therefore, we tried to isolate immunodominant proteins secreted by K. pneumoniae strains causing PLA.

Results: The secreted proteins of the NTUH-K2044 strain were separated by two-dimensional electrophoresis and then immunoblotted using convalescent sera from patients with $K$. pneumoniae PLA. A 30-kDa immunodominant protein was then identified. Liquid chromatography-tandem mass spectrometry (LC-MS/MS) revealed an open reading frame (KP1_p307) located on the pK2044 plasmid and bioinformatic analysis identified this protein as a signal peptide of unknown function. The KP1_p307 gene was more prevalent in PLA strains and capsular type $\mathrm{K} 1 / \mathrm{K} 2$ strains, but disruption of this gene in NTUH-K2044 strain did not decrease virulence in mice. Ten of fourteen (71\%) sera from patients with K. pneumoniae PLA were immunoreactive with the recombinant KP1_p307 protein. Seroconversion demonstrated by a rise in serum titer in serial serum samples confirmed that antibodies against the KP1_p307 protein were elicited after infection.
\end{abstract}

Conclusions: The KP1_p307 protein could be used as an antigen for early serodiagnosis of K. pneumoniae PLA, particularly in K1/K2 PLA strains.

Keywords: Klebsiella pneumoniae, Pyogenic liver abscess, Antigen, Serodiagnosis

\section{Background}

Klebsiella pneumoniae is a common Gram-negative enteric bacterium that causes hospital-acquired urinary tract infection, septicemia, and pneumonia as well as community-acquired pneumonia. Recently, communityacquired pyogenic liver abscess (PLA) caused by $K$. pneumoniae complicated with metastatic meningitis and endophthalmitis has emerged globally especially in Asia [1-13]. Based on analysis of data from the National Health Insurance Database in Taiwan, cases of PLA per year have increased from 1,950 in 1997 to 3,083 in 2008

\footnotetext{
* Correspondence: wangjt@ntu.edu.tw

'Department of Microbiology, National Taiwan University College of Medicine, 1, Sec 1, Jen-Ai Rd., Taipei, Taiwan

${ }^{6}$ Department of Internal Medicine, National Taiwan University Hospital, Taipei, Taiwan

Full list of author information is available at the end of the article
}

[14,15]. Among the 506 case-patients with PLA admitted to National Taiwan University Hospital (NTUH) from 2000 through 2004, 358 case-patients had positive culture results, and $286(79.9 \%)$ of these 358 case-patients had positive culture results showing $K$. pneumoniae infection [15]. In a nationwide prospective study of PLA in Korea, $K$. pneumoniae was the most common etiologic organism (78.2\%) [2]. K. pneumoniae was also reported as the most common etiological organism of PLA in New York, Hong Kong, Singapore, and Australia [2-11]. The mortality rates were $10 \%$ among those with $K$. pneumoniae-caused PLA and $3040 \%$ among those with metastatic meningitis. The survivors of meningitis usually had severe neurological sequelae and $K$. pneumoniae endophthalmitis usually resulting in blindness of the affected eyes.

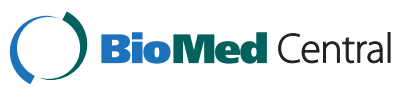

2014 Lin et al.; licensee BioMed Central. This is an Open Access article distributed under the terms of the Creative Commons Attribution License (http://creativecommons.org/licenses/by/4.0), which permits unrestricted use, distribution, and reproduction in any medium, provided the original work is properly credited. The Creative Commons Public Domain Dedication waiver (http://creativecommons.org/publicdomain/zero/1.0/) applies to the data made available in this article, unless otherwise stated. 
A previous study identified the predictors of septic metastatic infection and mortality among patients with K. pneumoniae liver abscess [16]. Most of the severe complications occurred within the first 3 days after hospital admission. Percutaneous drainage of primary liver abscess caused by K. pneumoniae lowered rates of mortality, metastatic infection, and complications. A previous study also observed that timing of appropriate antimicrobial therapy is a major determinant of survival and neurological outcome for patients with $K$. pneumoniae meningitis [17]. Therefore, rapid diagnosis and proper treatment for this invasive disease are required.

When a bacterial infection is suspected because of symptoms (such as fever) and increased white blood cell counts, the standard procedure in hospitals is to isolate and identify the bacterium. Identification of $K$. pneumoniae using automated or manual biochemical tests requires at least 24 to 48 hours. However, identification through molecular or immunologic techniques of bacterial nucleic acid and antigen detection can accelerate the diagnosis of bacterial infectious diseases. A realtime PCR assay with specific primers and hybridization probes targeting 16S rRNA gene was developed for the direct detection of $K$. pneumoniae from positive blood culture bottles [18]. But this method still requires culture enrichment.

In this study, we used a proteomic strategy to identify an immunodominant protein in $K$. pneumoniae strains causing PLA and evaluated the sensitivity and specificity of an immunoblot method for detecting antibodies to this antigen in patient sera.

\section{Methods}

\section{Ethics statement}

All the clinical bacterial strains used in this study were provided by the strain collection of National Taiwan University Hospital (NTUH) in Taiwan. The Ethics Committee approved that no formal ethical approval was needed to use these clinically obtained materials, because the strains were remnant from patient samples, and the data were analyzed anonymously. The collection and study of serum samples in this study was approved by the Institutional Review Board of NTUH and written informed consent was obtained from each participant (approval number: 200904046R). All animal experiments followed the guidelines in the Handbook of Laboratory Animal Care of the National Laboratory Animal Breeding and Research Center, National Science Council of Taiwan, and were approved by the Institutional Animal Care and Use Committee of the National Taiwan University College of Medicine (approval number: 20130005).

\section{Bacterial strains and culture conditions}

The $74 \mathrm{~K}$. pneumoniae clinical isolates used in this study were collected from NTUH from 1997 to 2003 [19]. Forty-two were PLA strains isolated from the blood of patients with PLA with or without meningitis or endophthalmitis complications, and 32 were non-tissueinvasive strains isolated from patients with sepsis but not PLA or other metastatic infections in any tissue. K. pneumoniae and Escherichia coli were cultured in LB medium at 37C supplemented with appropriate antibiotics, including $50 \mu \mathrm{g} / \mathrm{ml}$ kanamycin and $100 \mu \mathrm{g} / \mathrm{ml}$ ampicillin.

\section{Serum samples}

The serum samples from fourteen patients with $K$. pneumoniae PLA and three patients with non-K. pneumoniae liver abscess were obtained from NTUH in $2008 \sim 2010$. The dates of admission and sera collection were recorded. The diagnosis of liver abscess was confirmed by abdominal sonography or computed tomography, as well as isolation and identification of the pathogens from blood or abscess aspiration. The serum samples from twelve patients with $K$. pneumoniae infections (other than PLA) were obtained from Taipei Veterans General Hospital (VGH) in 2012. The diagnosis was based on clinical presentations and history.

\section{Purification of secreted proteins}

NTUH-K2044 strain was cultured overnight and pelleted by centrifugation. The culture supernatant was collected and $0.45-\mu \mathrm{m}$ filtered. The secreted proteins were precipitated by adding trichloroacetic acid to a final $20 \%$ concentration and incubating on ice for 30 minutes, collected after centrifugation, washed several times with cold acetone, air-dried, dissolved in $1.5 \mathrm{M}$ Tris- $\mathrm{Cl}$ ( $\mathrm{pH} 8.8$ ), and assayed to determine protein concentration using the Bradford method.

\section{Two-dimensional gel electrophoresis and immunoblotting} The proteins (2 $3 \mathrm{mg}$ ) were separated by two-dimensional electrophoresis. Briefly, protein extract in sample buffer (8 M urea, 2\% Pharmalyte pH 3 10, 60 mM DTT, 4\% CHAPS, bromophenol blue) was first separated on IPG strips (Immobiline DryStrip pH 3 10), then on $12 \%$ sodium dodecyl sulfate (SDS)-polyacrylamide gels (PAGE; $18 \mathrm{~cm} 20 \mathrm{~cm}$ ), and the separated proteins were transferred onto a poly(vinylidene) fluoride (PVDF) membrane (Millipore, Bedford, MA). The membrane was incubated with blocking buffer (5\% skimmed milk in PBS with $0.5 \%$ Tween 20 ) for $1 \mathrm{~h}$ at room temperature, primary antibody (sera from patients or healthy subjects or anti-GST antibody or anti-KP1_p307 antibody diluted in blocking buffer), secondary antibody (horseradish peroxidase [HRP]-conjugated goat anti-human IgG or 
HRP-conjugated goat anti-rabbit IgG [Chemicon, Temecula, CA]), and then enhanced chemiluminescence (ECL) reagent.

\section{Liquid chromatography-tandem mass spectrometry (LC-MS/MS) analysis}

The protein spots on the Coomassie blue stained twodimensional gel were excised and digested by trypsin. The peptide mixture was analyzed using a nanoAcquity system (Waters, Milford, MA) connected to an LTQOrbitrap Elite hybrid mass spectrometer (Thermo Fisher Scientific, Bremen, Germany) equipped with a nanospray interface (Proxeon, Odense, Denmark).

\section{Expression and purification of recombinant KP1_p307 protein}

KP1_p307 gene was amplified by PCR using primers KPP307-074F (5' -CCGTCGCCGGCGATAAATC-3') and KPP307-916R (5'-CGTAGATATCAGCGTTACCAAAG$\left.3^{\prime}\right)$ and then cloned into a pGEM-T easy plasmid. The KP1_p307 was sub-cloned into the Klenow fragment blunting-EcoRI site of a pGEX-4T-1 plasmid. The resulting plasmid was transformed into E. coli DH10B. KP1_p307 gene was amplified by PCR using primers KPP307-076F (5'-GTCGCCGGCGATAAATCCGG-3') and KPP307R (5' -TTATTCGTAGATATCAGCGTTACC-3') and cloned into the blunt-end SmaI site of a pQE32 plasmid. The resulting plasmid was transformed into E. coli M15/ pREP4. The recombinant GST-fused KP1_p307 protein and His-tagged KP1_p307 protein were expressed under $1 \mathrm{mM}$ isopropyl $\beta$-D-1-thiogalactopyranoside (IPTG) induction at $37 \mathrm{C}$ and purified per the manufacturer $\mathrm{s}$ instructions. The immuno-reactivities to GST-fused KP1_p307 protein and His-tagged KP1_p307 protein were not different. However, the yield of purified Histagged KP1_p307 protein was higher than that of purified GST-fused KP1_p307 protein because His-tagged protein (either in supernatant or in inclusion body) could be purified under denaturing condition. Therefore, we used the His-tagged KP1_p307 protein for further examination of the diagnostic application of KP1_p307 protein.

\section{Knockout of KP1_p307}

The KP1_p307 gene and its flanking regions were amplified by PCR using primers 307KO-F (5'-GTCCTGCTGAA ACATGACC- $\left.3^{\prime}\right)$ and 307KO-R (5'-ACACTATCGTGT CACAGATG-3'). Nucleotides 64902 of the KP1_p307 coding sequence were replaced by a kanamycin resistant gene. The deletion fragment was then cloned into the suicide vector pUT containing a spectinomycin resistant gene as a selective marker to distinguish between single and double crossovers for chromosomal integration [20,21]. The resulting plasmid was transferred to
NTUH-K2044 by conjugation. Chromosomal deletion of the KP1_p307 mutant was confirmed by PCR with different primer pairs.

\section{Virulence assay in mice}

Female 5-week-old BALB/cByl mice were inoculated with $K$. pneumoniae intraperitoneally as previously described [22]. Four mice were used to test the effects of each inoculum. After inoculation, the mice were observed for 30 days. The LD50 was calculated using the method established by Reed and Muench. For in vivo competition, the KP1_p307 mutant strain and the fully virulent plac $Z$ deletion mutant strain in the logarithmic phase were mixed at a $1: 1$ ratio $\left(\begin{array}{ll}1 & 10^{3}\end{array}\right.$ colony-forming units each) in saline, and inoculated intraperitoneally into eight 5-week-old BALB/c mice as previously described [23]. The amounts of bacteria in livers and spleens one day after inoculation were determined by plating serial dilutions onto LB agar containing $1 \mathrm{mmol} / \mathrm{L}$ IPTG and $50 \mathrm{mg} / \mathrm{mL}$ X-gal. In vivo competition was assessed using the competitive index (the output to input ratio of the KP1_p307 mutant strain [blue colony] to the placZ deletion mutant strain [white colony]).

\section{Immunoblotting of recombinant KP1_p307 protein with patient sera}

The purified recombinant His-tag KP1_p307 protein was separated on $12 \%$ SDS-PAGE ( $~ 5$ ng per lane) and then transferred onto a membrane. Patients sera (1:2000 dilution) were screened simultaneously using Mini-PROTEAN II Multiscreen Apparatus (Bio-Rad, Hercules, CA). Those collected first were screened first, and immunoblotting with serial sera was performed when the first collected serum was antibody negative.

\section{Capsular typing by wzc sequencing}

Capsular types of the K. pneumoniae strains were determined by $w z c$ genotyping [24]. The sequences of CD1VR2-CD2 region were compared with those of reference strains by NCBI-nucleotide blast and the corresponding capsular types were determined.

\section{Results}

\section{Identification of immunodominant proteins in} NTUH-K2044 strain

Proteins released by bacteria to the extracellular environment are usually the antigens that elicit immune responses, have diagnostic value, and can provide protective immunity. To identify immunodominant proteins in K. pneumoniae NTUH-K2044 strain causing pyogenic liver abscess (PLA), the secreted proteins were isolated as described above and analyzed by immunoblotting using sera from one healthy subject and two patients in the convalescent phase of PLA caused by $K$. pneumoniae 


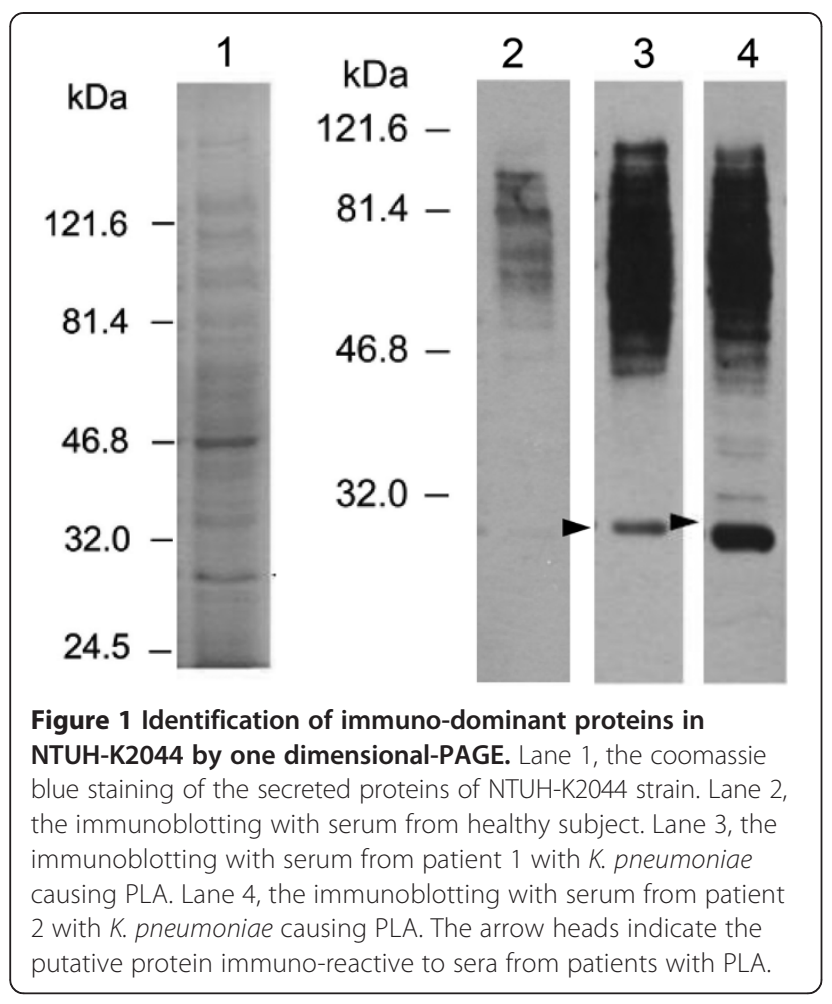

(Figure 1). The convalescent sera from patients with K. pneumoniae PLA were primarily used to identify immunodominant proteins secreted by $K$. pneumoniae strains causing PLA. Because sera obtained from patients in the convalescent stage were considered to be especially rich in antibodies against the infectious agent. A $30-\mathrm{kDa}$ band was recognized by the sera from the two patients but not the serum from the healthy subject. Further separation by two-dimensional gel electrophoresis and immunoblotting with the above sera followed by Coomassie blue staining (Figure 2) identified a similar band (approximately $30-\mathrm{kDa}$ molecular weight and isoelectric point [pI] ranging from 7 to 9) that was immunoreactive with patients sera and therefore a possible candidate protein for immunological diagnosis of PLA caused by $K$. pneumoniae.

\section{Identification of the protein immunoreactive to sera from patients with PLA}

The protein spots on the two-dimensional gels were excised, digested by trypsin, and analyzed by LC-MS/MS. The obtained peptide sequences were compared with the whole genome sequences of NTUH-K2044 strain (chromosome and a large plasmid: AP006725.1 and AP006726.1). A 921-bp open reading frame (KP1_p307) located on the large plasmid pK2044 was confirmed. The sequences of peptides obtained from LC-MS/MS represented $93.1 \%$ of KP1_p307 protein sequences. The theoretical pI and molecular weight of KP1_p307 protein (i.e., 8.87 and 32,254.72-Da) matched those of the immunoreactive protein. The amino acid sequences of KP1_p307 revealed 47\% (107/229) identity to a hypothetical protein of Lactobacillus rossiae (WP_026017099.1) and 53\% (95/180) identity to a hypothetical protein of Enterococcus dispar (WP_016173221.1). Bioinformatic
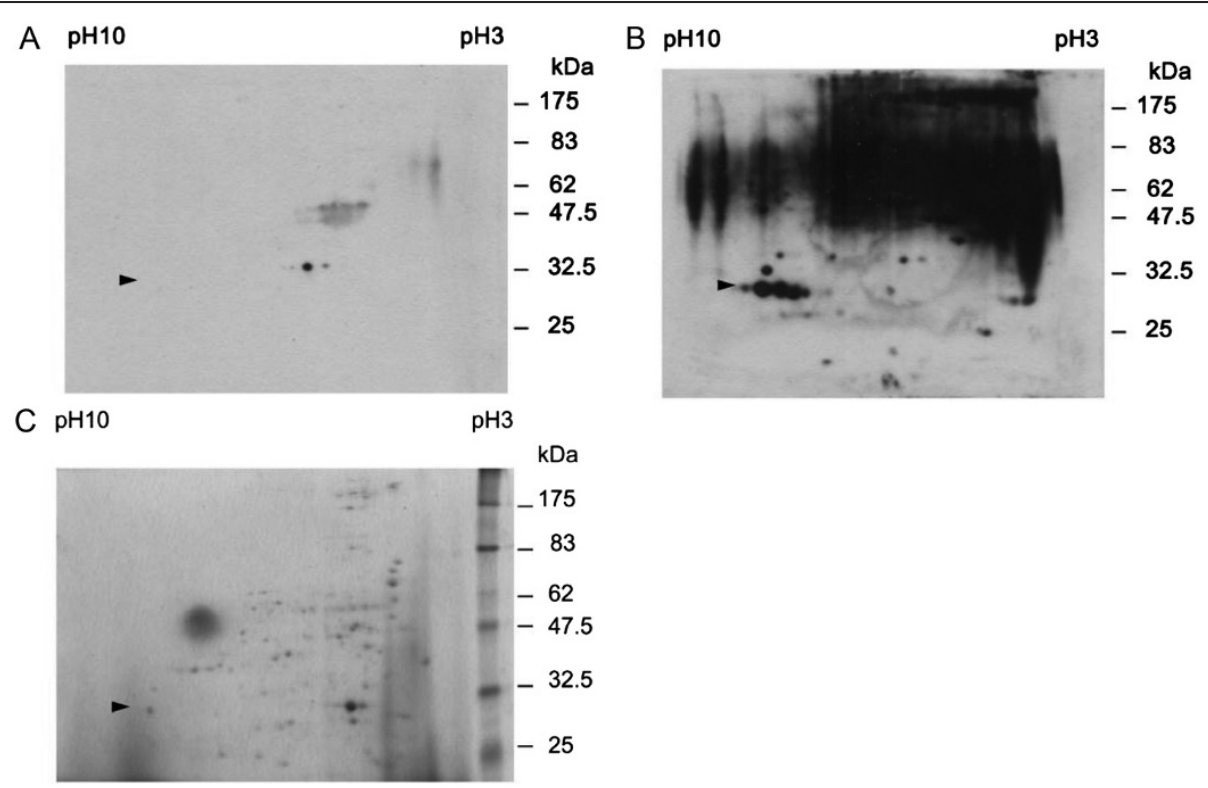

Figure 2 Identification of immuno-dominant proteins in NTUH-K2044 by two dimensional-gel electrophoresis. A, the immunoblotting with serum from healthy subject. B, the immunoblotting with serum from patient 2 with K. pneumoniae causing PLA. $\mathbf{C}$, the coomassie blue staining of the secreted proteins of NTUH-K2044 strain. The arrow heads indicate the putative protein immuno-reactive to sera from patients with PLA. 
analysis revealed that this protein contained a signal peptide located at $\mathrm{N}$-terminal residues 1 to 24 but had no known function.

\section{Confirmation of the immunoreactivity of KP1_p307 protein}

The recombinant GST-KP1_p307 protein was expressed and purified in $E$. coli to confirm its immunoreactivity. Sera from another four patients with PLA and three healthy subjects were used to immunoblot the recombinant GST-KP1_p307 protein. The GST-KP1_p307 protein was immunoreactive with sera from patients but not from healthy subjects (Figure 3 ). The immunoreactive signal of the secreted proteins disappeared when the immunoreactive serum was pre-incubated with the recombinant GST-KP1_p307 protein but not with GST protein (data not shown). These results confirmed that KP1_p307 protein was the candidate protein identified above.

\section{Prevalence of strains with KP1_p307}

The prevalence of PLA strains and non-tissue invasive strains with KP1_p307 was determined by PCR and Western blotting using rabbit anti-KP1_p307 serum (Table 1). The results of PCR and Western blotting

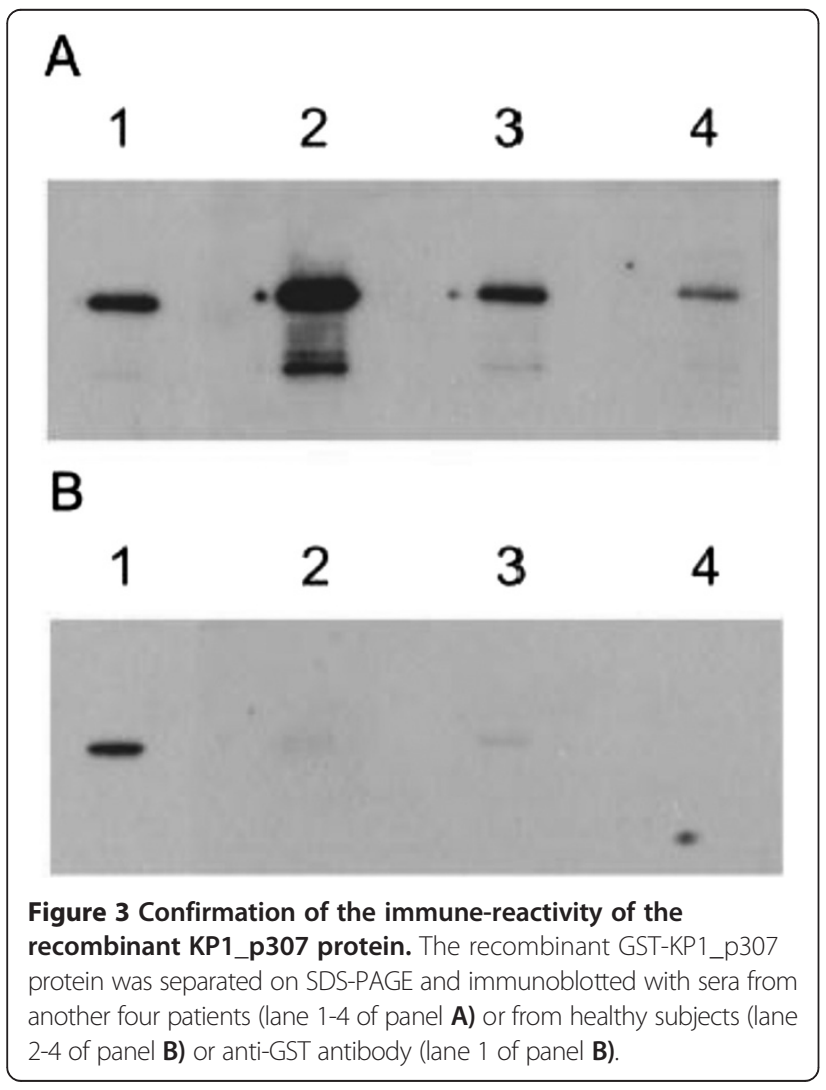

were consistent. The prevalence of PLA strains with KP1_p307 (39 out of 42) was significantly higher than that of non-tissue invasive strains with KP1_p307 (6 out of 32) (Chi-square analysis, $P<0.0001$ ). K1 and K2 were the most prevalent capsular types causing PLA and were strongly associated with virulence. Thirtyseven of the 42 PLA strains were $\mathrm{K} 1 / \mathrm{K} 2$, whereas 4 of the 32 non tissue-invasive strains were found to be K1/ $\mathrm{K} 2$. The prevalence of K1/K2 strains with KP1_p307 (39 out of 41) was significantly higher than that of non-K1/ K2 strains with KP1_p307 (6 out of 33) (Chi-square analysis, $P<0.0001)$. Because this gene might be associated with bacterial virulence, a KP1_p307 knockout mutant of the NTUH-K2044 strain was generated and its virulence was tested in mice. The survival of mice intraperitoneally infected with $110^{3} \mathrm{cfu}$ of wild type or KP1_p307 knockout mutant was not significantly different (Figure 4A). Knockout of KP1_p307 did not increase the lethal dose (LD50 $<110^{3} \mathrm{cfu}$ ) of NTUHK2044 strain given intraperitoneally. Moreover, the in vivo competition between the wildtype and KP1_p307 knockout mutant strains was also not significantly affected (Figure 4B).

\section{Diagnostic application of KP1_p307 protein in $K$. pneumoniae PLA}

Sera from 14 patients with $K$. pneumoniae PLA were collected to evaluate the diagnostic application of KP1_p307 protein (Table 2). Ten of the 14 (71.4\%) sera were immunoreactive with the recombinant KP1_p307 protein. Because not all the PLA strains have KP1_p307, the isolates from the four patients whose sera did not react to the recombinant $\mathrm{KP} 1$ _p307 protein were tested to determine whether they possess KP1_p307. Two out of these four strains did not have KP1_p307, and therefore could not elicit these antibodies. Moreover, sera from three patients with LA not caused by $K$. pneumoniae and sera from ten healthy subjects were not immunoreactive with the recombinant KP1_p307 protein. Therefore, presence of antibodies against the KP1_p307 protein can be implicated in $K$. pneumoniae PLA.

Antibodies against the KP1_p307 could be detected in the first serum sample (collected 126 days after admission) from eight of the ten patients with positive reactions. Four of these eight patients had positive serological reactions to KP1_p307 protein on the first day after hospital admission (Table 2). The other two patients (I and J) were observed to seroconvert (Figure 5) on the $5^{\text {th }}$ and $10^{\text {th }}$ day after admission, respectively, and to still have anti-KP1_p307 antibody 4 months after infection (on the $110^{\text {th }}$ day). These results confirm that the antibodies against the KP1_p307 protein were elicited after infections. Therefore, the KP1_p307 protein could be used to serodiagnose $K$. pneumoniae PLA. 
Table 1 The prevalence of KP1_p307 among 42 PLA strains and 32 non-tissue invasive strains

\begin{tabular}{llllll}
\hline & \multicolumn{2}{l}{ PLA strains $(\mathbf{n}=\mathbf{4 2})$} & & \multicolumn{2}{c}{ Non-tissue invasive strains $(\mathbf{n}=\mathbf{3 2})$} \\
\cline { 2 - 3 } \cline { 5 - 6 } & K1/K2 & non-K1/K2 & & K1/K2 & non-K1/K2 \\
\hline KP1_P307+ & 35 & 4 & 4 & 2 \\
KP1_P307 - & 2 & 1 & 0 & 26 \\
\hline
\end{tabular}

The prevalence of KP1_P307: PLA strains vs. non-tissue invasive strains $(P<0.0001$, Chi-square analysis); K1/K2 strains vs. non-K1/K2 strains $(P<0.0001$, Chi-square analysis).

\section{Diagnostic application of KP1_p307 protein in $K$. pneumoniae infections other than PLA}

The diagnostic application of KP1_p307 protein in $K$. pneumoniae infections other than PLA was further examined (Table 3). Sera from 12 non-PLA patients were obtained from VGH and their $K$. pneumoniae isolates were also collected. The capsular type was determined by $w z c$ genotyping and the presence of KP1_p307 was determined by PCR. The three strains with K1 or K2 capsular type all possess KP1_p307 and five out of the 9 non-K1/K2 strains (55.6\%) have KP1_p307. Four of the 8 sera $(50 \%)$ collected from patients infected with KP1_p307-positive K. pneumoniae strains when they visited the hospital were immunoreactive with the recombinant KP1_p307 protein.

\section{Discussion}

In this study, we identified an immunodominant antigen in a K. pneumoniae PLA strain by a proteomic approach and evaluated its application to the serodiagnosis of $K$. pneumoniae PLA. The sensitivity of $K$. pneumoniae PLA detection was $71.4 \%(10 / 14)$ and four out of six sera (66.7\%) collected on the first day of hospitalization for K. pneumoniae PLA were positive for anti-KP1_p307 antibodies. Our previous study indicated that $80 \%$ of patients with PLA were caused by $K$. pneumoniae infection [15]. Therefore, the case of non-K. pneumoniae PLA was relatively few. We included serum samples from three patients with LA not caused by $K$. pneumoniae and sera from ten healthy subjects to confirm the specificity. The results indicated that the specificity was $100 \%(13 / 13)$. The detection of antibodies against KP1_p307 protein could be performed directly using sera and did not require the cultivation of bacteria. Although the sensitivity and specificity of this test still need to be confirmed by using a larger number of clinical samples, we suggest that detection of anti-KP1_p307 antibody was considered to be an easy and rapid method for early screening of $K$. pneumoniae PLA. As shown in Figure 5, patient I was observed to still have anti-KP1_p307 antibody 4 months after infection (on the $110^{\text {th }}$ day), therefore antibody titers could persisted for months after successful
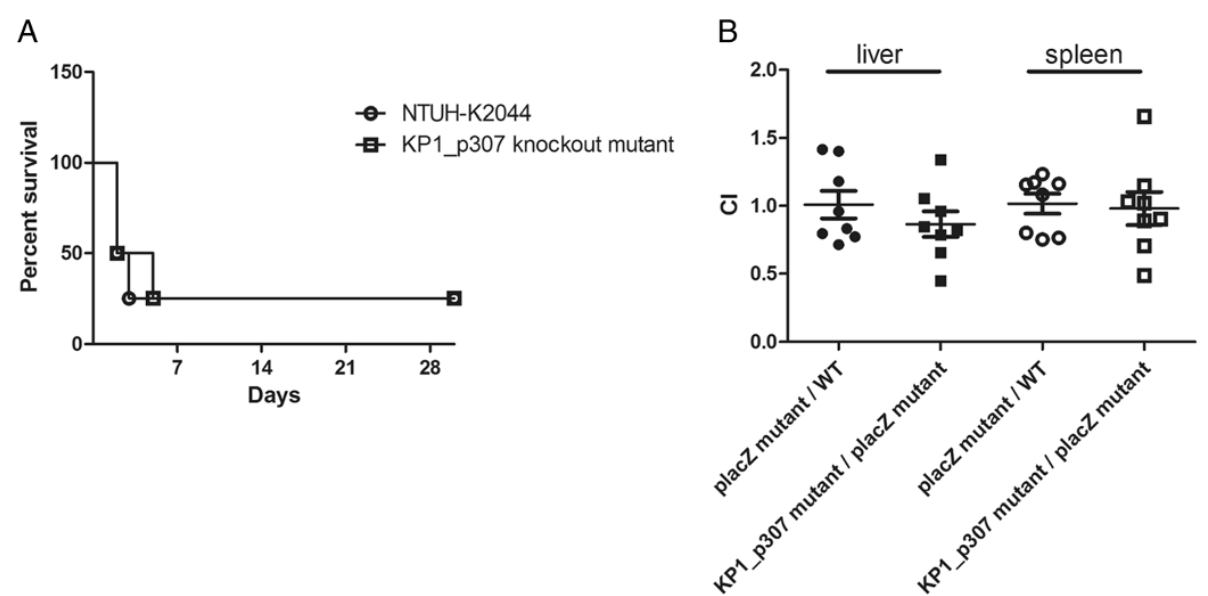

Figure 4 The virulence of KP1_p307 knockout mutant. A, The survival curve of mice with intraperitoneal infection of wild type or KP1_p307 knockout mutant. Female 5-week-old BALB/CByl mice were inoculated with $110^{3} \mathrm{cfu}$ of $K$. pneumoniae wild type or KP1_p307 knockout mutant intraperitoneally (four mice each group). The survival of mice infected with wild type or KP1_p307 knockout mutant was not significantly different ( $P=0.870$, log-rank test). B, The in vivo competition between wild type and KP1_p307 mutant in liver and spleen of BALB/C mice. The wild type strain (blue colony on LB agar containing IPTG and X-gal) and the placZ deletion mutant (white colony) or KP1_p307 mutant strain (blue colony) and the placZ deletion mutant strain (white colony) were mixed at a 1:1 ratio and inoculated intraperitoneally into eight BALB/C mice. The amounts of bacteria in livers and spleens in the next day after inoculation were determined. The in vivo competition index (Cl) was calculated. The Cl of lacZ mutant/WT in liver is 1.0090 .101 and that of KP1_p307 mutant/lacZ mutant in liver is 0.8650 .094 . The Cl of lacZ mutant/WT in spleen is 1.0160 .073 and that of KP1_p307 mutant/ lacZ mutant in spleen is 0.9810 .121 . The in vivo competition of wild type and KP1_p307 mutant was not significantly different $(P=0.315$ in liver, $P=0.808$ in spleen, Student s t test). 
Table 2 The immuno-reaction to KP1_p307 protein of sera from 17 patients with liver abscess

\begin{tabular}{|c|c|c|c|c|c|c|c|c|}
\hline \multirow{2}{*}{$\begin{array}{l}\text { Patients } \\
\text { A }\end{array}$} & \multirow{2}{*}{$\begin{array}{l}\text { Diagnosis } \\
\text { K. pneumoniae causing PLA }\end{array}$} & \multicolumn{6}{|c|}{ Serum collection day (the day after admission) } & \multirow{2}{*}{$\begin{array}{l}\text { KP1_p307 PCF } \\
N D\end{array}$} \\
\hline & & 3 & & & & & & \\
\hline B & & 1 & & & & & & ND \\
\hline C & & 1 & & & & & & ND \\
\hline $\mathrm{D}$ & & 26 & & & & & & ND \\
\hline E & & 1 & & & & & & ND \\
\hline $\mathrm{F}$ & & 4 & & & & & & ND \\
\hline G & & 4 & & & & & & ND \\
\hline $\mathrm{H}$ & & 1 & & & & & & ND \\
\hline । & & 3 & 5 & 7 & 110 & & & ND \\
\hline J & & 2 & 4 & 10 & 14 & 25 & 39 & ND \\
\hline K & & 2 & 26 & & & & & + \\
\hline L & & 1 & 7 & 22 & & & & + \\
\hline$M$ & & 3 & 7 & 55 & & & & - \\
\hline N & & 1 & 6 & 14 & & & & - \\
\hline $\mathrm{O}$ & PLA (non-Kp) & 5 & 30 & 60 & & & & \\
\hline$P$ & PLA (non-Kp) & 1 & 11 & & & & & \\
\hline Q & amoebic LA & 1 & 13 & & & & & \\
\hline
\end{tabular}

The number in boldface indicates the positive reaction to KP1_p307 protein and the number in standard indicates the negative reaction to KP1_p307 protein. ND: not determined.

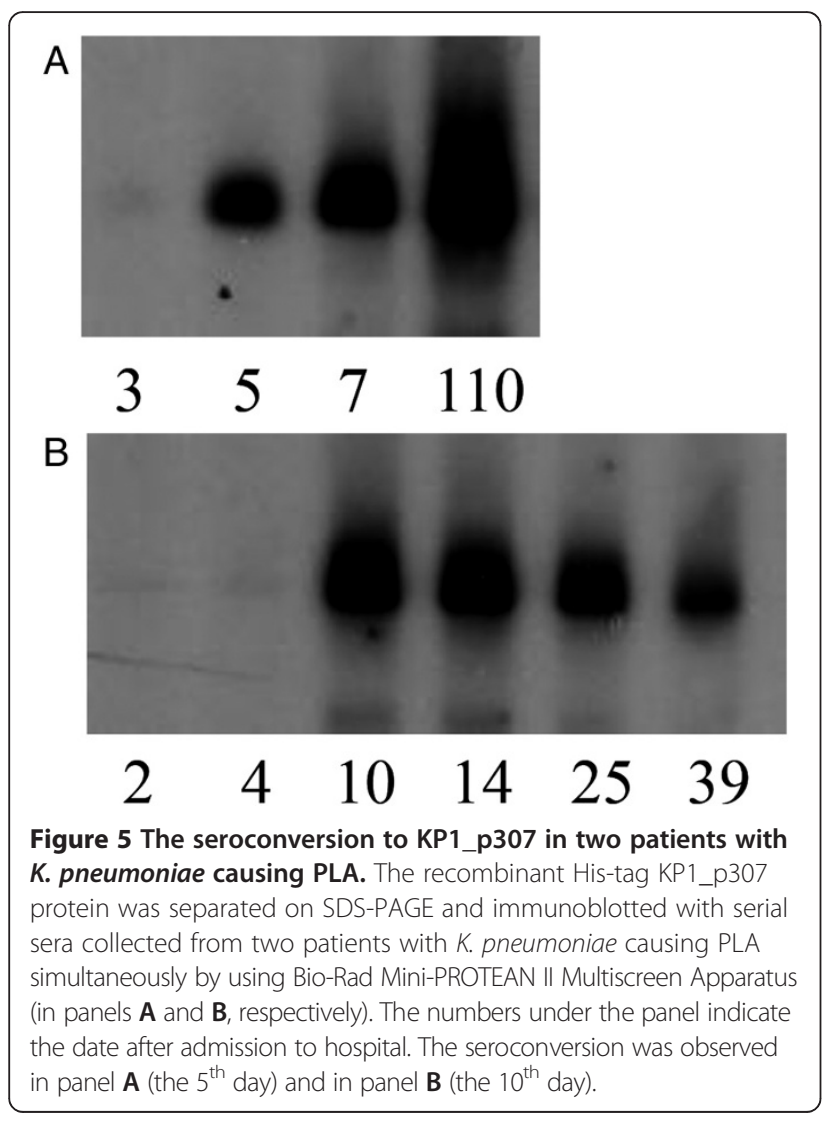

treatment. If the antibodies persist, the KP1_p307 antigen could not be used in the diagnosis of $K$. pneumoniae re-infection. However, because we did not have adequate follow-up sera, how long the anti-KP1_p370 antibodies persisted could not be determined.

The KP1_p307 protein was predicted to have a signal peptide similar to that of the KP1_p307 protein present in the fraction secreted from bacteria. The amino acid sequences of KP1_p307 revealed approximately 50\% identity to a hypothetical protein of $L$. rossiae and a hypothetical protein of $E$. dispar. Hence, the crossreactivity of antibodies against $L$. rossiae and $E$. dispar to KP1_p307 protein may occur. However, the infections caused by $L$. rossiae and $E$. dispar were extremely rare. There were neither $L$. rossiae nor $E$. dispar isolates in the bacteria collection of NTUH, Taipei VGH and Chang Gung Memorial Hospital, thus we have difficulties to examine the crossreactivity. But, we expected that the possible crossreactivity would not interfere in the application of KP1_p307 to the serodiagnosis of K. pneumoniae PLA. Moreover, the function of KP1_p307 protein remains unknown and difficult to study. Although the presence of this protein was correlated with $K$. pneumoniae PLA, knock out of KP1_p307 did not decrease the virulence of K. pneumoniae NTUH-K2044 strain in mice even its in vivo ability to compete with wild type strain. Our preliminary result demonstrated that the anti-KP1_p307 antibodies could not block the infection of $K$. pneumoniae NTUH-K2044 strain in mice (data 
Table 3 The immuno-reaction to KP1_p307 protein of sera from the 12 patients with K. pneumoniae infections (other than PLA)

\begin{tabular}{|c|c|c|c|c|}
\hline Patients & Diagnosis & Capsular type $^{1}$ & KP1_p307 PCR & KP1_p307 immunoreaction \\
\hline 1 & primary bacteremia & $\mathrm{K} 2$ & + & + \\
\hline 2 & necrotizing fasciitis with bacteremia & $\mathrm{K} 2$ & + & - \\
\hline 3 & primary bacteremia & $\mathrm{K} 1$ & + & - \\
\hline 4 & necrotizing fasciitis & K62 & + & + \\
\hline 5 & primary bacteremia & K58 & + & - \\
\hline 6 & renal abscess with bacteremia & K5 & + & + \\
\hline 7 & primary bacteremia & K74 & - & - \\
\hline 8 & cholecystitis with bacteremia & K58 & - & - \\
\hline 9 & cholecystitis with bacteremia & K46 & - & - \\
\hline 10 & urinary tract infection & K58 & + & + \\
\hline 11 & pneumonia with bacteremia & K68 & + & - \\
\hline 12 & primary bacteremia & $\mathrm{K} 28$ & - & - \\
\hline
\end{tabular}

${ }^{1}$ Capsular types of these strains were determined by using wzc genotyping.

not shown). Further investigation of the functions of KP1_p307 protein and anti-KP1_p307 antibody is still needed.

Previous studies have demonstrated the correlation of the virulence o f $K$. pneumoniae $\mathrm{K} 1$ strains with the presence of aerobactin [25,26]. Our previous study also reported that p-rmpA gene (for regulator of mucoid phenotype A) was associated with $K$. pneumoniae PLA [27]. However, knockout of the iuc region (which encodes aerobactin) or p-rmpA did not decrease its virulence in mice $[27,28]$. Here, the KP1_p307 was also associated with $K$. pneumoniae PLA, but knockout of this gene had no influence on virulence. Because these genes (iuc region, p-rmpA and KP1_p307) are all located on the large plasmid and associated with bacterial virulence, we propose that other virulence factors might be present in the large plasmid of $K$. pneumoniae. These genes could serve as markers of bacterial virulence due to their co-inheritance together with virulence genes carried by this plasmid.

As shown in Table 1, the presence of KP1_p307 was also correlated with capsular type $\mathrm{K} 1 / \mathrm{K} 2$. The $K$. pneumoniae strains with capsular types $\mathrm{K} 1$ and $\mathrm{K} 2$ are more virulent than those with other capsular types [25,29]. Therefore, the KP1_p307 can be used to detect not only K. pneumoniae PLA but also possibly other infections caused by capsular type $\mathrm{K} 1 / \mathrm{K} 2$ strains. The majority of K. pneumoniae PLA strains were capsular type $\mathrm{K} 1$ and K2 [19]. But we did not obtain all the K. pneumoniae strains isolated from these 14 PLA patients in this study (Table 2); consequently the capsular types of these isolates were unknown. Besides PLA, the diagnostic application of KP1_p307 protein in K. pneumoniae other infections was also examined. All strains with $\mathrm{K} 1$ or $\mathrm{K} 2$ capsular type possess KP1_p307 gene and 55.6\% of non-
K1/K2 strains also have KP1_p307. But only $50 \%$ of sera collected from patients infected with KP1_p307-positive $K$. pneumoniae strains were immunoreactive with KP1_p307 protein. Because these sera samples were collected at the first day when the patients visited the hospital, the anti- KP1_p307 antibodies may not yet be elicited at this very early time point. These results demonstrated that the detection of anti-KP1_p307 antibody implicated the infections by several capsular types (particularly $\mathrm{K} 1$ and $\mathrm{K} 2$ ) of $K$. pneumoniae which carry a virulent plasmid.

There are several immunogenic antigens of $K$. pneumoniae, such as OmpA and OmpK36 etc., have been reported previously [30]. The immuno-reactivity to OmpK36 of sera obtained from PLA patients was also examined. But the sera samples from all the K. pneumoniae PLA patients were not immuno-reactive to the recombinant OmpK36 protein (data not shown). We speculated that OmpK36 antigen located on the outer membrane might be masked by capsule and might not be exposed, because the strains causing PLA were observed to be heavily encapsulated [22]. The antibodies against OmpK36 might be produced at a later stage or might not be elicited during $K$. pneumoniae PLA infections. Moreover, OmpK36 protein is commonly distributed among $K$. pneumoniae strains and has sequence similarity to that of $E$. coli. These results indicated that KP1_p307 would be a better antigen than OmpK36 to serodiagnose K. pneumoniae PLA.

\section{Conclusions}

In conclusion, an immunodominant antigen, KP1_p307, was identified in a $K$. pneumoniae strain causing PLA. The KP1_p307 gene was located on the large plasmid and more prevalent in PLA strains and capsular type 
K1/K2 strains, but deletion of KP1_p307 did not decrease the virulence of this strain in mice. The high sensitivity and specificity demonstrated here indicates that the Kp1_p307 protein could be used to serodiagnose K. pneumoniae PLA or other infections.

\section{Competing interests}

The authors declare that they have no competing interests.

\section{Authors contributions}

All authors participated in the design and interpretation of the studies. LTL wrote the manuscript. WJT reviewed the manuscript. All authors read and approved the final manuscript.

\section{Acknowledgements}

This work was supported by grants funding from National Science Council, National Taiwan University, National Taiwan University Hospital, and the Liver Disease Prevention and Treatment Research Foundation in Taiwan.

\section{Author details}

'Department of Microbiology, National Taiwan University College of Medicine, 1, Sec 1, Jen-Ai Rd., Taipei, Taiwan. ${ }^{2}$ Department of Microbiology and Immunology, National Defense Medical Center, Taipei, Taiwan.

${ }^{3}$ Department of Internal Medicine, Far Eastern Memorial Hospital, New Taipei City, Taiwan. ${ }^{4}$ Division of Infectious Diseases, Department of Medicine, Taipei Veterans General Hospital, Taipei, Taiwan. ${ }^{5}$ School of Medicine, National Yang-Ming University, Taipei, Taiwan. ${ }^{6}$ Department of Internal Medicine, National Taiwan University Hospital, Taipei, Taiwan.

Received: 5 August 2014 Accepted: 11 December 2014

Published online: 21 December 2014

\section{References}

1. Chang SC, Fang CT, Hsueh PR, Chen YC, Luh KT: Klebsiella pneumoniae isolates causing liver abscess in Taiwan. Diagn Microbiol Infect Dis 2000, 37(4):279 284

2. Chung DR, Lee SS, Lee HR, Kim HB, Choi HJ, Eom JS, Kim JS, Choi YH, Lee JS, Chung MH, Kim YS, Lee H, Lee MS, Park CK, Korean Study Group for Liver Abscess: Emerging invasive liver abscess caused by K1 serotype Klebsiella pneumoniae in Korea. J Infect 2007, 54(6):578 583.

3. Fang FC, Sandler N, Libby SJ: Liver abscess caused by magA+ Klebsiella pneumoniae in North America. J Clin Microbiol 2005, 43(2):991 992.

4. Karama EM, Willermain F, Janssens X, Claus M, Van den Wijngaert S, Wang JT, Verougstraete C, Caspers L: Endogenous endophthalmitis complicating Klebsiella pneumoniae liver abscess in Europe: case report. Int Ophthalmol 2008, 28(2):111 113.

5. Keynan Y, Karlowsky JA, Walus T, Rubinstein E: Pyogenic liver abscess caused by hypermucoviscous Klebsiella pneumoniae. Scand J Infect Dis 2007, 39(9):828 830.

6. Kohayagawa Y, Nakao K, Ushita M, Niino N, Koshizaki M, Yamamori Y, Tokuyasu Y, Fukushima H: Pyogenic liver abscess caused by Klebsiella pneumoniae genetic serotype K1 in Japan. J Infect Chemother 2009, 15(4):248 251

7. Liew KV, Lau TC, Ho CH, Cheng TK, Ong YS, Chia SC, Tan CC: Pyogenic liver abscess a tropical centres experience in management with review of current literature. Singap Med J 2000, 41(10):489 492.

8. Lok KH, Li KF, Li KK, Szeto ML: Pyogenic liver abscess: clinical profile, microbiological characteristics, and management in a Hong Kong hospital. J Microbiol Immunol Infect 2008, 41(6):483 490.

9. Moore R, O Shea D, Geoghegan T, Mallon PW, Sheehan G: Communityacquired Klebsiella pneumoniae liver abscess: an emerging infection in Ireland and Europe. Infection 2013, 41(3):681-686.

10. Nadasy KA, Domiati-Saad R, Tribble MA: Invasive Klebsiella pneumoniae syndrome in North America. Clin Infect Dis 2007, 45(3):e25 e28.

11. Pastagia M, Arumugam V: Klebsiella pneumoniae liver abscesses in a public hospital in Queens, New York. Travel Med Infect Dis 2008, 6(4):228 233.

12. Rahimian J, Wilson T, Oram V, Holzman RS: Pyogenic liver abscess: recent trends in etiology and mortality. Clin Infect Dis 2004, 39(11):1654 1659.
13. Siu LK, Yeh KM, Lin JC, Fung CP, Chang FY: Klebsiella pneumoniae liver abscess: a new invasive syndrome. Lancet Infect Dis 2012, 12(11):881 887.

14. Lin YT, Liu CJ, Chen TJ, Chen TL, Yeh YC, Wu HS, Tseng CP, Wang FD, Tzeng $\mathrm{CH}$, Fung CP: Pyogenic liver abscess as the initial manifestation of underlying hepatocellular carcinoma. Am J Med 2011, 124(12):1158 1164.

15. Tsai FC, Huang YT, Chang LY, Wang JT: Pyogenic liver abscess as endemic disease, Taiwan. Emerg Infect Dis 2008, 14(10):1592 1600.

16. Lee SS, Chen YS, Tsai HC, Wann SR, Lin HH, Huang CK, Liu YC: Predictors of septic metastatic infection and mortality among patients with Klebsiella pneumoniae liver abscess. Clin Infect Dis 2008, 47(5):642 650

17. Fang $C T$, Chen YC, Chang SC, Sau WY, Luh KT: Klebsiella pneumoniae meningitis: timing of antimicrobial therapy and prognosis. QJM 2000, 93(1):45 53

18. Kurupati P, Chow C, Kumarasinghe G, Poh CL: Rapid detection of Klebsiella pneumoniae from blood culture bottles by real-time PCR. J Clin Microbiol 2004, 42(3):1337 1340.

19. Chuang YP, Fang CT, Lai SY, Chang SC, Wang JT: Genetic determinants of capsular serotype $\mathrm{K} 1$ of Klebsiella pneumoniae causing primary pyogenic liver abscess. J Infect Dis 2006, 193(5):645 654

20. Chou HC, Lee CZ, Ma LC, Fang CT, Chang SC, Wang JT: Isolation of a chromosomal region of Klebsiella pneumoniae associated with allantoin metabolism and liver infection. Infect Immun 2004, 72(7):3783 3792.

21. Ma LC, Fang $C T$, Lee $C Z$, Shun $C T$, Wang JT: Genomic heterogeneity in Klebsiella pneumoniae strains is associated with primary pyogenic liver abscess and metastatic infection. J Infect Dis 2005, 192(1):117 128.

22. Fang $C T$, Chuang YP, Shun $C T$, Chang SC, Wang JT: A novel virulence gene in Klebsiella pneumoniae strains causing primary liver abscess and septic metastatic complications. J Exp Med 2004, 199(5):697 705.

23. Hsieh PF, Lin HH, Lin TL, Wang JT: CadC regulates cad and tdc operons in response to gastrointestinal stresses and enhances intestinal colonization of Klebsiella pneumoniae. J Infect Dis 2010, 202(1):52 64.

24. Pan YJ, Lin TL, Chen YH, Hsu CR, Hsieh PF, Wu MC, Wang JT: Capsular types of Klebsiella pneumoniae revisited by wzc sequencing. PLoS One 2013, 8(12):e80670.

25. Nassif $X$, Sansonetti PJ: Correlation of the virulence of Klebsiella pneumoniae $\mathrm{K} 1$ and $\mathrm{K} 2$ with the presence of a plasmid encoding aerobactin. Infect Immun 1986, 54(3):603 608.

26. Yu WL, Ko WC, Cheng KC, Lee CC, Lai CC, Chuang YC: Comparison of prevalence of virulence factors for Klebsiella pneumoniae liver abscesses between isolates with capsular $\mathrm{K} 1 / \mathrm{K} 2$ and non-K1/K2 serotypes. Diagn Microbiol Infect Dis 2008, 62(1):1 6.

27. Hsu CR, Lin TL, Chen YC, Chou HC, Wang JT: The role of Klebsiella pneumoniae $\mathrm{mpA}$ in capsular polysaccharide synthesis and virulence revisited. Microbiology 2011, 157(Pt 12):3446 3457.

28. Hsieh PF, Lin TL, Lee CZ, Tsai SF, Wang JT: Serum-induced iron-acquisition systems and TonB contribute to virulence in Klebsiella pneumoniae causing primary pyogenic liver abscess. J Infect Dis 2008, 197(12):1717 1727.

29. Yeh KM, Kurup A, Siu LK, Koh YL, Fung CP, Lin JC, Chen TL, Chang FY, Koh TH: Capsular serotype K1 or K2, rather than magA and rmpA, is a major virulence determinant for Klebsiella pneumoniae liver abscess in Singapore and Taiwan. J Clin Microbiol 2007, 45(2):466 471.

30. Kurupati $P$, Teh BK, Kumarasinghe G, Poh CL: Identification of vaccine candidate antigens of an ESBL producing Klebsiella pneumoniae clinical strain by immunoproteome analysis. Proteomics 2006, 6(3):836 844

doi:10.1186/s12866-014-0321-4

Cite this article as: Lin et al:: Identification of an immuno-dominant protein from Klebsiella pneumoniae strains causing pyogenic liver abscess: implication in serodiagnosis. BMC Microbiology 2014 14:321. 